\title{
The association between self-rated health and different anthropometric and body composition measures in the Chinese population
}

\author{
Kun Tang ${ }^{1 *+}$, Yingxi Zhao ${ }^{2+}$ and Chunyan $\mathrm{Li}^{3}$
}

\begin{abstract}
Background: To analyze the strength of association between self-rated health and six anthropometric and body composition measures to explore the best indicator.

Methods: Analyses were based on the cross-sectional data from the China Kadoorie Biobank Study and approximately 300,000 adults were analyzed. Logistics regression was used to analyze the association between self-rated health (good or poor) and anthropometric and body composition measures (height, weight, body mass index (BMI), waist circumference (WC), hip circumference (HC) and body fat percentage, waist-to-hip ratio and waist-to-height ratio). Stratified analyses were undertaken to understand the effect modification of socioeconomic status on the association.

Result: Odds ratio of self-rated better health had an inverted U-shape association with weight, BMI, WC and body fat, with weight levels increasing until around 73.8 and $65.7 \mathrm{~kg}$ for male and female, BMI around $26.8 \mathrm{~kg} / \mathrm{m}^{2}$, WC around 85.8 and $87.6 \mathrm{~cm}$, body fat around 24.3 and $36.3 \%$, and then declining thereafter. Height and HC also indicated a slightly inverted U-shape association. The strongest association was observed after adjustment was weight, with one standard deviation greater weight associated with $10.2 \%$ and 10.6\% increased odds in male and female.

Conclusions: Being underweight and overweight are both risk factors for poor self-rated health in males and females, and weight is the best indicator of self-rated health compared with other measures.
\end{abstract}

Keywords: Self-rated Health, Obesity, BMI, Waist Circumference, Chinese

\section{Background}

Self-rated health has been proved to be a valid and reliable indicator for overall morbidity [1] and a good predictor of mortality $[2,3]$. Determinants of self-rated health include a great range of domains: sociodemographic factors including age [4] and employment [5, 6]; diagnosed chronic health conditions [7]; psychological factors [8]; and health behaviors [7], among which anthropometric measure of BMI has been considered an important indicator [8-15].

Past research analyzing the relation between overweight/obesity and self-rated health indicated that

\footnotetext{
* Correspondence: tangkun@hsc.pku.edu.cn

${ }^{\dagger}$ Equal contributors

'Department of Global Health, Peking University, Beijing, China

Full list of author information is available at the end of the article
}

people with overweight and obesity reported poor selfrated health more often than those of normal weight [8-15]. However, most of those studies focused on the association with body mass index (BMI) or percentage body fat [15], and failed to include other important anthropometric and body composition measures, including height, weight, waist circumference, hip circumference, waist-to-hip ratio (WHR) and waist-toheight ratio (WHtR), some of which proved to be better indicators of body adiposity [16-18].

Moreover, little attention has been given to whether the association between these anthropometric and body composition measures and self-rated health varies across population groups of different socioeconomic status and demographic characteristics. While the research on selfrated health and anthropometric and body composition 
measures is limited in China, most of those studies of selfrated health focused on socioeconomic aspects [19-21].

This study aims to analyze the strength of association between self-rated health and eight anthropometric and body composition measures (i.e. height, weight, BMI, waist circumference, hip circumference, body fat, WHR and WHtR) in China to explore the best indicator, and whether the strength association varies by different socioeconomic status and demographic characteristics The result of this study could provide public health practitioners an insight of what anthropometric indicators should be used to target peoples of various socioeconomic and demographic groups to achieve maximum effectiveness and efficiency.

\section{Methods}

\section{Study design and population}

The present study is based on the data requested from the China Kadoorie Biobank (CKB) study, which is a prospective cohort study of chronic disease in China. Details of the study design and characteristics of the study participants have been described previously [22, 23]. Briefly, 512,891 participants without major disabilities living in administrative units (rural villages or urban residential committees), aged 30-79 years (mean age: 51.5 years), were recruited in the baseline survey from five urban (Harbin, Qingdao, Suzhou, Liuzhou, Haikou) and five rural areas (from Henan, Hunan, Sichuan, Gansu and Zhejiang) in China between 2004 and 2008. The survey sites were selected based on geographic location, population stability, quality of death and disease registries, and local commitment and capacity. Within each site, permanent residents in each of the 100-150 administrative units (rural villages or urban residential committees) that were selected for the study were identified from local records and sent a letter or leaflet inviting them to participate. The participation rate was $33 \%$ in rural areas and $27 \%$ in urban areas.

To minimize of effect of existing disease conditions and using the "healthy" population for analyses, the current study excluded 172,373 participants (73,938 males and 98,435 females) who have self-reported the following major diseases, including diabetes, coronary artery heart disease, stroke or transient ischemic attack, hypertension, rheumatic heart diseases, tuberculosis, emphysema/bronchitis, asthma, cirrhosis/chronic hepatitis, peptic ulcer, gallstone/gallbladder diseases, kidney disease, fracture, rheumatoid arthritis, psychiatric disorders, neurasthenia, head injury and cancer. Besides, the analyses also excluded people (5903 males and 7664 females) who were at the extremes of the eight anthropometric and body composition measures (i.e. $>99.5$ percentile or $<0.5$ percentile of the distribution for all eight measures) to limit effects of any possible measurement error. A total of 326,951 adults (130,418 males and 196,533 females) formed the sample for the present analyses.

Ethical approval was obtained from the University of Oxford, the China National Center for Disease Control and Prevention (CDC) and the institutional review boards at the local CDCs in the 10 regions before the start of the survey. Written informed consent was obtained from all participants.

\section{Measures and variables}

A standardized questionnaire was administered by trained interviewers at the baseline survey using a laptop-based data-entry system, with built-in functions to prevent logical errors and missing values. Questions included socioeconomic and demographic status, health condition and medical history, behavioral pattern including smoking, drinking, physical activity and diet. In terms of physical measurements, trained staffs across the 10 survey sites conducted the standardized measurements with a protocol and instrument. All of the utilized devices were regularly maintained and calibrated for consistency in measurements.

Eight main anthropometric and body composition measures variables, either directly measured or derived, were assessed as exposure variables, including standing height, weight, body mass index (BMI), waist circumference (WC), hip circumference (HC), body fat percentage, waist-to-hip ratio (WHR) and waist-to-height ratio (WHtR). Standing height was measured to the nearest $0.1 \mathrm{~cm}$ with an audiometer. Weight was measured to the nearest $0.1 \mathrm{~kg}$ using a body composition analyzer (TANITA-TBF-300GS; Tanita Corporation), with subtraction of weight of clothing $(0.5 \mathrm{~kg}$ in summer, $1.0 \mathrm{~kg}$ in spring/autumn and $2.0-2.5 \mathrm{~kg}$ in winter). BMI was calculated as the weight in kilograms divided by the square of the height in meters $\left(\mathrm{kg} / \mathrm{m}^{2}\right)$. WC and $\mathrm{HC}$ were measured to the nearest $0.1 \mathrm{~cm}$ with a soft no stretchable tape. WC was measured mid-way between the lowest rib and the iliac crest or, when this was not practicable, $1 \mathrm{~cm}$ above the umbilicus (usually against bare skin in both cases, but subtracting $1 \mathrm{~cm}$ if on top of undergarments). $\mathrm{HC}$ was measured at the maximum circumference around the buttocks (usually over underpants, but subtracting $1 \mathrm{~cm}$ if over a skirt, or $2.5 \mathrm{~cm}$ if over trousers). Body fat percentage was estimated by the Tanita body composition analyzer using proprietary algorithms, which reflects the fraction of total weight that was estimated to be fat weight. WHR and WHtR were calculated using the above measures. The issues of quality control (QC) was mentioned in one of the reference paper. At the baseline, QC survey data were available for 15,728 participants $(3.1 \%)$, with the mean length of time 
between baseline and QC survey being 17 days [standard deviation $(\mathrm{SD})=36$ days]. There was good agreement between the baseline and QC survey for several common variables. The height, weight and BMI showed extremely high correlation with baseline measures $(0.99,0.96$ and 0.93 , respectively), whereas for other measures of adiposity (waist and hip circumferences, and body fat percentage), they ranged from 0.82 to 0.90 .

Each exposure variables were also classified into several categories for the analyses: Height was categorized into 7 groups (threshold 157, 160, 163, 166, 169 and $172 \mathrm{~cm}$ for male; $146,150,153,156,159,162 \mathrm{~cm}$ for female), weight into 8 and 7 groups (threshold 52, 56, 60, 64, 68, 72 and $76 \mathrm{~kg}$ for male; 48, 52, 56, 60, 64 and $68 \mathrm{~kg}$ for female), BMI into 8 groups (threshold 18, 20, $22,24,26,28,30 \mathrm{~kg} / \mathrm{m} 2$ ), WC into 8 groups (threshold 72, 76, 80, 84, 88, 92 and $96 \mathrm{~cm}$ for male; 66, 70, 74, 78, 82,86 and $90 \mathrm{~cm}$ for female), HC into 7 groups (threshold $82,85,88,91,94$ and $97 \mathrm{~cm}$ for male; 83, 86, 89, 92, 95 and 98 for female), body fat into 7 groups (14, 17, 20, 23, 26 and $29 \%$ for male; 23, 26, 29, 32, 35 and $38 \%$ for female), WHR into 7 groups $(0.82,0.85,0.88,0.91$, 0.94, 0.97 for male; $0.79,0.82,0.85,0.88,0.91,0.94$ for female), WHtR into 7 groups $(0.42,0.45,0.48,0.51$, 0.54, 0.57).

In this study, the outcome variable was self-rated health, which was an ordinal variable that rated general health on a 4-point rating scale ranging from "excellent" to "poor." Respondents were asked, "How is your current general health status?" Responses were "excellent," "good," "fair," or "poor". For those who reported "good" and above were coded as "1", "poor" and "fair" were coded as "0". The dichotomies data was then included in the analyses and " 0 " was used as the reference group.

Other covariates included study area, sex, age category (in decile), the highest education completed (i.e. no formal school, primary school, middle school, high school, or college/university), household income last year in Chinese yuan (i.e. <2500, 2500-4999, 5000-9999, 10,000-19,999, 20,000-34,999, >35,000), marital status (i.e. married, widowed, separated/divorced, never married), smoking status (i.e. never, occasional, former, or current regular), alcohol consumption (i.e. never, occasional, former, or current regular), and total physical activity in metabolic equivalent hours per day (METhours/day) [24].

\section{Statistical analyses}

Selected demographic, socioeconomic, behavioral and anthropometric characteristics were described separately for male and female. The distribution of sociodemographic, unadjusted means with standard deviations (SD) for continuous variables and unadjusted proportions for categorical variables were presented.
The association between each anthropometric and body composition measure and self-rated health was analyzed using logistics regression. Odds ratios (OR) of self-rated good health were reported within each category of the anthropometric and body composition measures, adjusting for the covariates, including study area, age category, education level, household income level, marital status, smoking status, alcohol consumption, and MET-hours/day. To analyze the association between per standard increase and the self-reporting better health, each exposure measures were divided by the SD of that particular variable and entered the regression as a continuous variable. ORs of self-rated health were regressed on levels of each anthropometric measure as a continuous variable, with adjustment for covariates as described above.

To evaluate the effect modification of socioeconomic and demographic factors, stratified analyses were performed using logistics regression, and ORs were analyzed in each stratum of socioeconomic and demographic factors. All statistical analyses were performed using SAS version 9.3 (SAS Institute Inc., Cary, North Carolina, USA), and tests results were reported significant at 0.05 levels.

\section{Results}

Baseline participant characteristics stratified by sex are presented in Table 1. Overall, of the 326,951 participants included in the analyses, 130, 418 (39.9\%) were men, and 132,297 (40.5\%) resided in urban areas. At the time of the survey, the mean age was $50.74 \pm 10.57$ years for male and $48.85 \pm 9.96$ years for female. More men (58.4\%) than women (45.3\%) finished middle school or above. Both the prevalence of current smoking $(64.2 \%$ compared with $2.1 \%$ ) and weekly drinking (34.7\% vs. $2.1 \%)$ were higher among men than women. Men were also more physically active (25.4 compared than women with 21.7 MET-hours/day). Compared with men, women had lower height (154.3 vs. $165.3 \mathrm{~cm})$, lower weight (56.1 vs. $63.6 \mathrm{~kg})$, slightly higher BMI (23.5 vs. $23.2 \mathrm{~kg} /$ $\mathrm{m} 2)$ and larger $\mathrm{HC}(90.7$ vs. $90.2 \mathrm{~cm})$ but lower WC $(78.0$ vs. $81.1 \mathrm{~cm})$ and higher body fat $(31.6$ vs. $21.7 \%)$. 71,817 male $(55.1 \%)$ and 96,585 female $(49.1 \%)$ reported their self-rated health as "excellent" or "good".

Figure 1 shows the sex-specific associations between self-rated health and anthropometric and body composition measures. The OR had an inverted U-shape association with weight, BMI, WC and body fat, with weight levels increasing until around 73.8 and $65.7 \mathrm{~kg}$ respectively for male and female, BMI level around 26.8, WC level around 85.8 and 87.6, body fat around 24.3 and 36.3 , and then declining thereafter. Height, $\mathrm{HC}$ and WHtR also showed a slightly inverted U-shape 
Table 1 Basic Characteristics of 326,951 Participants by Sex

\begin{tabular}{|c|c|c|}
\hline & $\begin{array}{l}\text { Male } \\
(N=130418)\end{array}$ & $\begin{array}{l}\text { Female } \\
(N=196533)\end{array}$ \\
\hline \multicolumn{3}{|l|}{ Age, years (\%) } \\
\hline $30-39$ & 16.83 & 20.15 \\
\hline $40-49$ & 31.38 & 35.35 \\
\hline $50-59$ & 29.87 & 28.80 \\
\hline $60-69$ & 16.20 & 12.04 \\
\hline$\geq 70$ & 5.72 & 3.66 \\
\hline \multicolumn{3}{|l|}{ Education (\%) } \\
\hline Illiterate & 8.65 & 23.37 \\
\hline Elementary & 32.96 & 31.35 \\
\hline Middle school & 33.73 & 27.09 \\
\hline High school & 17.67 & 13.85 \\
\hline College and University & 7.00 & 4.33 \\
\hline \multicolumn{3}{|l|}{ Household Income (\%) } \\
\hline$<4,999$ yuan & 9.29 & 9.70 \\
\hline $5,000-9,999$ yuan & 17.88 & 20.53 \\
\hline $10,000-19,999$ yuan & 28.83 & 30.20 \\
\hline 20,000-34,999 yuan & 25.06 & 24.03 \\
\hline$\geq 35,000$ yuan & 18.93 & 15.54 \\
\hline \multicolumn{3}{|l|}{ Marital Status (\%) } \\
\hline Married & 93.16 & 90.86 \\
\hline $\begin{array}{l}\text { Divorced, widowed, separated } \\
\text { or never married }\end{array}$ & 6.84 & 9.14 \\
\hline \multicolumn{3}{|l|}{ Tobacco use (\%) } \\
\hline Nonsmoker & 14.01 & 95.68 \\
\hline Occasional smoker & 11.47 & 1.69 \\
\hline Former smoker & 10.35 & 0.58 \\
\hline Regular smoke & 64.17 & 2.05 \\
\hline \multicolumn{3}{|l|}{ Alcohol use (\%) } \\
\hline Never regular drinker & 19.00 & 61.78 \\
\hline Ex regular drinker & 2.19 & 0.27 \\
\hline Occasional or seasonal drinker & 33.47 & 33.99 \\
\hline Monthly drinker & 6.91 & 1.50 \\
\hline Reduced intake & 3.69 & 0.37 \\
\hline Weekly & 34.74 & 2.10 \\
\hline $\begin{array}{l}\text { Physical activity, MET-hours/day } \\
\text { (mean } \pm \text { SD) }\end{array}$ & $23.53(15.29)$ & $21.66(12.92)$ \\
\hline \multicolumn{3}{|l|}{ Province (\%) } \\
\hline Qingdao (Urban) & 8.35 & 6.64 \\
\hline Harbin (Urban) & 9.03 & 9.54 \\
\hline Haikou (Urban) & 5.31 & 6.89 \\
\hline Suzhou (Urban) & 9.34 & 8.86 \\
\hline Liuzhou (Urban) & 7.60 & 9.07 \\
\hline Sichuan (Rural) & 10.74 & 11.54 \\
\hline Gansu (Rural) & 10.94 & 11.49 \\
\hline
\end{tabular}

Table 1 Basic Characteristics of 326,951 Participants by Sex (Continued)

\begin{tabular}{lll}
\hline Henan (Rural) & 14.31 & 13.27 \\
Zhejiang (Rural) & 11.05 & 10.76 \\
Hunan (Rural) & 13.32 & 11.93 \\
$\begin{array}{l}\text { Anthropometric and body } \\
\text { composition measures (mean } \pm \text { SD) }\end{array}$ & & \\
Height (cm) & $165.27(6.23)$ & $154.33(5.71)$ \\
Weight (kg) & $63.59(9.77)$ & $56.12(8.59)$ \\
BMl (kg/m²) & $23.22(2.97)$ & $23.52(3.12)$ \\
Waist Circumference (cm) & $81.13(8.90)$ & $78.02(8.58)$ \\
Hip Circumference $(\mathrm{cm})$ & $90.27(6.26)$ & $90.70(6.18)$ \\
Body Fat (\%) & $21.72(5.80)$ & $31.56(6.51)$ \\
Waist to Hip Ratio & $0.90(0.05)$ & $0.86(0.07)$ \\
Waist to Height Rario & $0.49(0.06)$ & $0.51(0.06)$ \\
\hline
\end{tabular}

association. There was a slightly inverted U-shape for WHR in male, but a positive linear association in female.

Figures 2 shows OR of self-reported good health associated in per 1 sex-specific SD of each anthropometric and body composition measure, adjusting for other socioeconomic, demographic and behavioral covariates. In both sexes, the single strongest anthropometric indicator after adjusting for all confounders was weight, with each 1 SD greater weight associated with OR of $1.102(95 \%$ CI 1.088-1.117) and 1.106 (95\%CI 1.095-1.117) in male and female. The next strongest predictor was BMI, with OR of 1.096 (95\%CI 1.080-1.110) for male and 1.090 (95\%CI 1.080-1.101) for female, while height was the weakest predictors, with only 1.029 (95\%CI 1.015-1.042) for male and 1.052 (95\%CI 1.041-1.063) for female.

Table 2 shows the effect modification of self-rated health OR among different socioeconomic strata. Education level and household income appeared to be the strongest modifier in affecting self-rated health and anthropometric measures, while the difference of the association between self-rated health and anthropometric measures among different age group and region group (rural or urban) was weaker in significance. In our adjusted model, male with an income level between 5000 and 19,999 yuan were more likely to report better selfrated health with increase in weight than male with income above 20,000 (OR 1.131, 95\%CI 1.110-1.153; vs. OR 1.080, 95\% CI 1.059-1.101), while when the income level was above 20,000 yuan or education level above high school, the association between increase in anthropometric measures and increase in self-rated health was weaker. Such association was insignificant in female. 

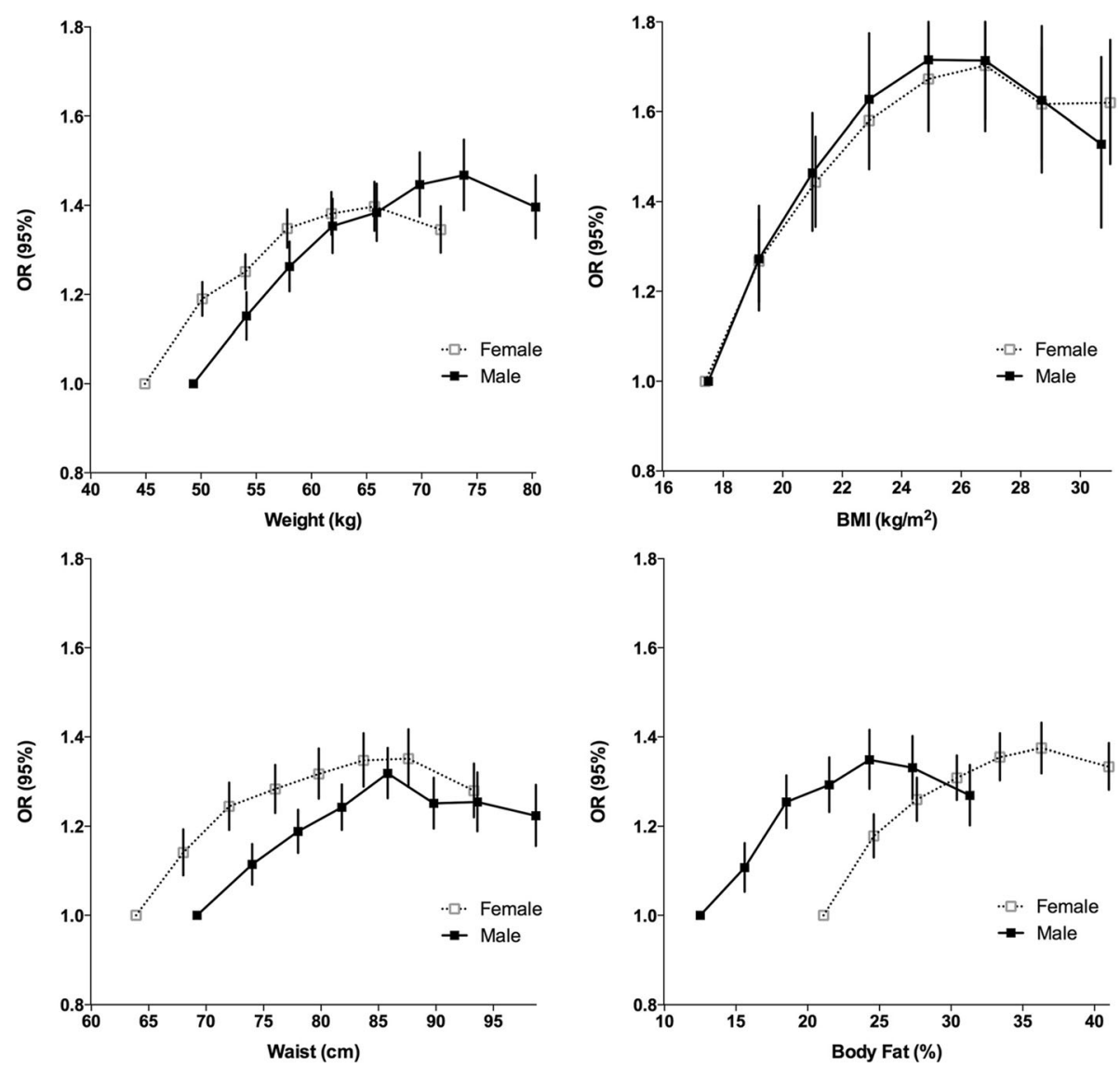

Fig. 1 Sex-specific associations between self-rated health and 4 anthropometric and body composition measures, adjusted for study area, age category, education level, household income level, marital status, smoking status, alcohol consumption, and MET-hours/day

\section{Discussion}

This study is the first and also the largest scale research in China concerning the strength of association between self-rated health and anthropometric and body composition measures. One of the major findings was that weight was most strongly associated with self-reporting good health, while BMI was also a good indicator. Further analyses also indicated that the association could be modified by sex, income and education level. The association between self-rated health and anthropometric and body composition measures appeared relatively stronger among male with less income and lower education, while weaker among female.

There are several potential limitations of the present study: firstly, in the present analysis, we excluded patients with all major chronic preconditions, which covered a wide range of 19 diseases (e.g. cardiovascular disease, diabetes, hypertension, etc.). Nonetheless we only excluded the participants with self-reported diseases, and the population may not be regarded as completely "healthy". . Secondly, the study used Tanita body composition analyzer, which was originally developed and validated only in Japanese populations. Although little evidence suggests there's significant difference among East Asian populations like the Chinese and Japanese, in terms of body fat composition. It could potentially create a systematic bias in the analysis. Thirdly, the study population was selected from the baseline data on a cohort study and the healthy volunteer effect may exist. The population might be more sensitive to health issues and more likely to report bad health. However, the large sample size and excluding patients with major diseases shall reduce the effect to minimal. Lastly, the participation rate of the study is relatively low. While the low response rate is a problem for population based prevalence study, our study, which is drawn from the baseline of a large cohort study, does not mean to represent the whole Chinese population. Moreover, like in the U.S. Nurses' Health Study [25] and the Health Professionals Study [26], a particular study population with strong homogeneity could already generate important associations with strong clinical and public health implications.

The inverted U-shaped associations between the four anthropometric and body composition measures (weight, BMI, WC and body fat) and self-rated health 


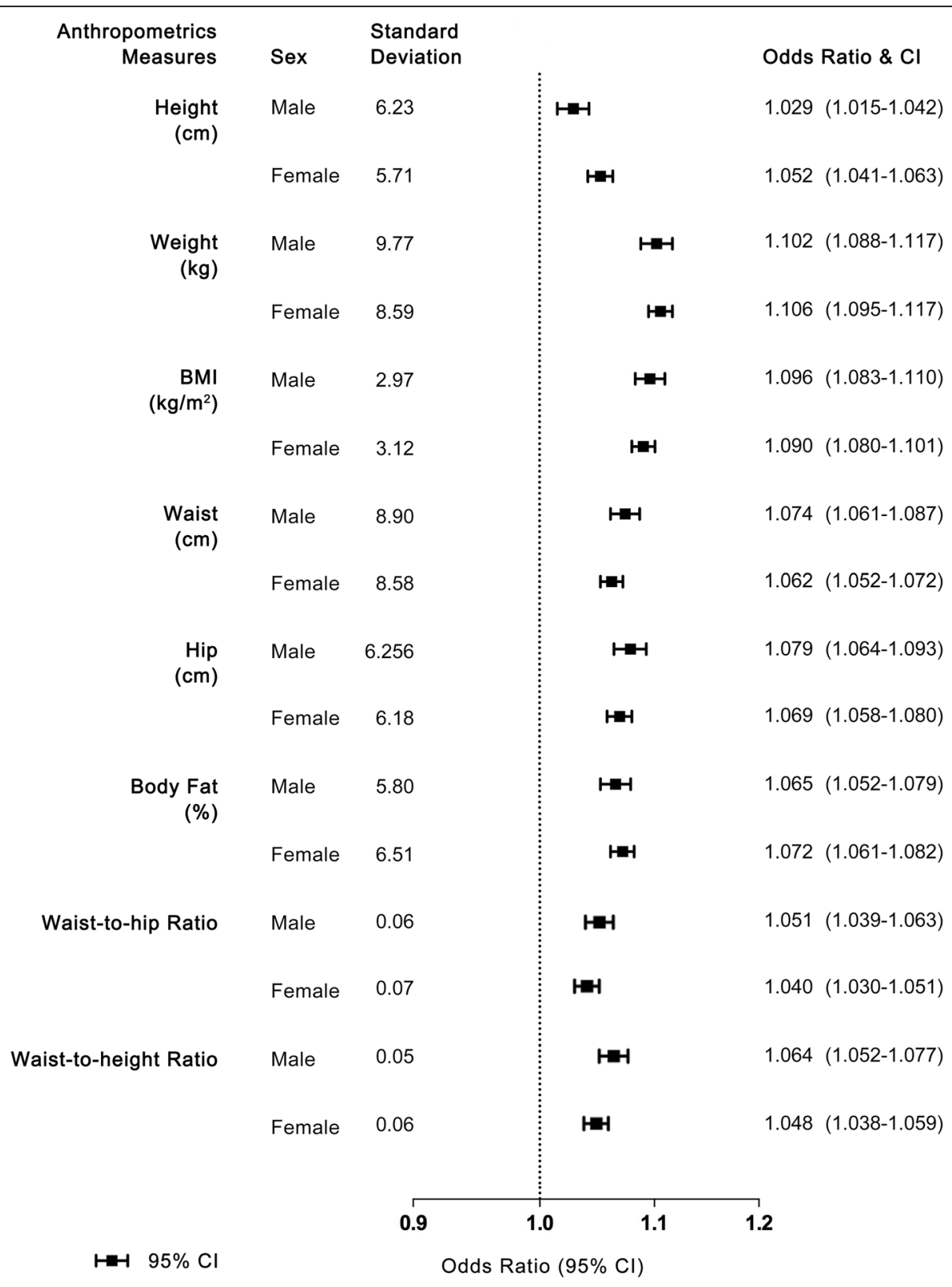

Fig. 2 Odds ratio of self-reported good health associated with per 1 sex-specific SD of each anthropometric and body composition measure, adjusted for study area, age category, education level, household income level, marital status, smoking status, alcohol consumption, and MET-hours/day

found in this study indicated that both overweight and underweight were associated with poor self-rated health. Being underweight (lower weight, BMI, body fat and WC) might be considered malnourished which indicates bad health in Chinese people's traditional opinions, while the stigma of obesity is growing globally as obesity rates rise, thus weight, BMI, WC and body fat being too high might also give people indirect effect of obesity in limiting their functional capacity, and also an ill feeling mentally. This is the first analysis of the association between BMI and self-rated health in China, and the findings were consistent with those previously reported by several smaller studies $[9,11,14,27]$ but differ from those in several other studies, which indicated that only being overweight was associated with poor self-rated health [28], or in some cases did not discuss underweight [12, 15]. The reasons for these inconsistencies are uncertain, but this study is larger than any previous studies. One possible explanation is that all the participants in the study are middle-aged to elderly Chinese, and there might be possible societal or cultural differences regarding body image and health with other population [13]. 


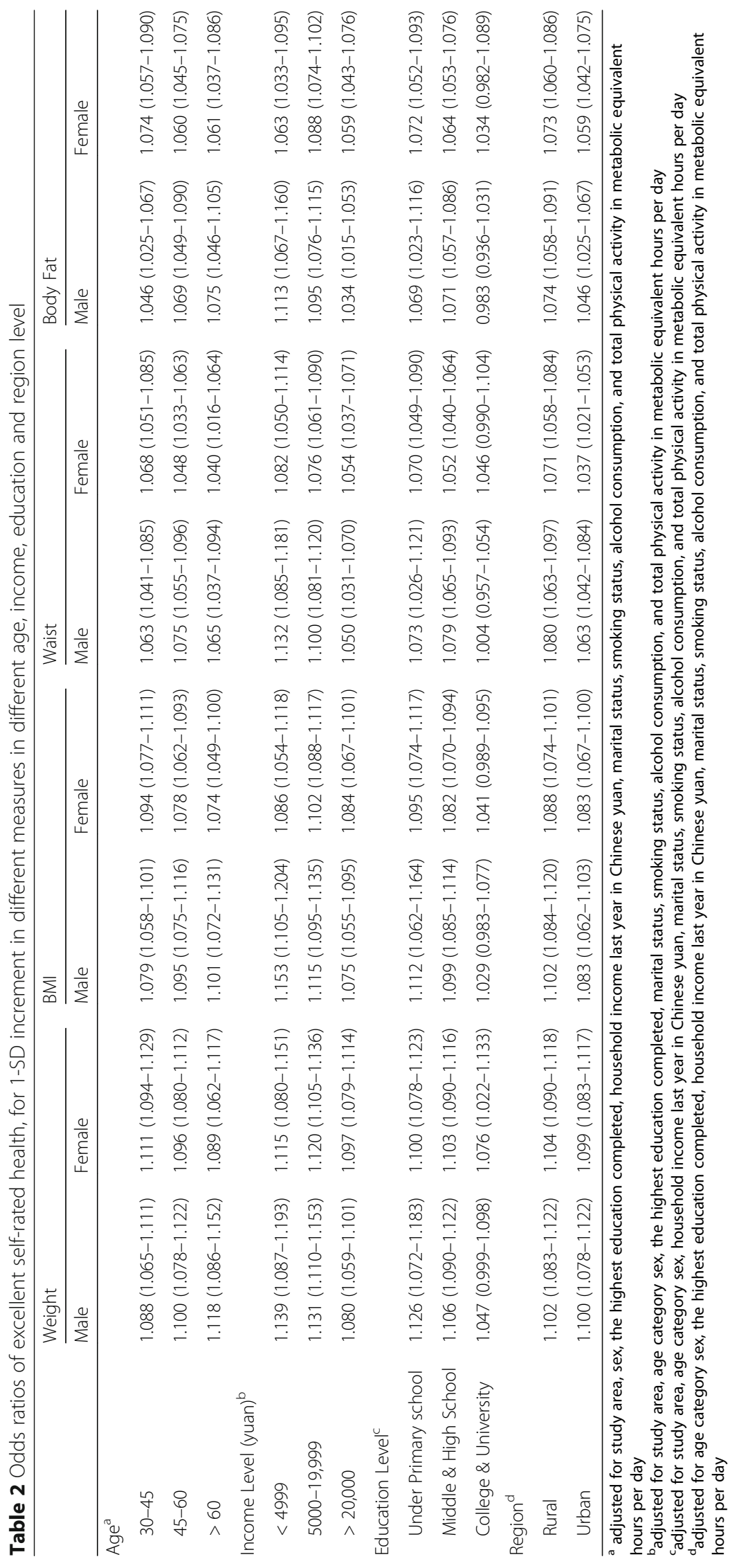


Moreover, unlike most former studies, our main analyses also excluded participants with major diseases, which enable us to explore the unbiased association between anthropometric and body composition measures and self-rated health.

Our findings suggested a positive linear association between WHR and self-rated health in females but not males. This is different to the associations with other anthropometric indices. However, whether the same result can be observed in other studies of the Chinese population still needs to be validated. Further more, if such observation is indeed true, further studies should be conducted to understand the underlying physiological or psychological mechanisms.

Our study also found that weight and BMI are better indicators than WC and WHR, suggesting that general adiposity might be a better indicator of self-rated health than central or regional adiposity. The 1-SD increase of weight and WC could be considered similar effect on adiposity, as past studies have established that a $3 \mathrm{~kg}$ increase in weight was corresponded to approximately $3 \mathrm{~cm}$ increase in WC [29], indicating that the superiority of weight and BMI to WC is due to their direct effect on self-rated health. One possible explanation could be that people are more sensitive to change in weight than WC, as weight is easier for daily measurement and $\mathrm{WC}$ is a variable that seldom used in daily life.

In the further analyses, we found that the strength of association between self-rated health and anthropometric and body composition measures differs among socioeconomic status especially education level and household income. While middle-income and lower educated male tended to have the strongest influence with increase in adiposity to increase in self-rated health, one possible explanation is that the group of higher education level and income level has overall better selfrated health as explained in several former studies [28]. This group with higher education level has more access to health knowledge and better health seeking behavior, thus is less influenced by anthropometric and body composition measures. The population with lower education level and less household income has poorer selfrated health on average, and is more strongly affected by change in the measures. Our study also found that the association was weaker among female, and this may be due to the fact that almost all female face greater societal pressures, such as weight discrimination and body image problems [30, 31], regardless of socioeconomic status.

\section{Conclusion}

The current study describes the strength of association between self-rated health and anthropometric and body composition measures. It demonstrates that being underweight and overweight are both risk factors for poor self-rated health in males and females in the Chinese populations. Further analyses also indicate that general adiposity is better associated with self-rated health than central adiposity, and the association between self-rated health and anthropometric and body composition measures are modified by sex, income and education level. Additional research is needed to study other anthropometric and adiposity measures' strength of influence on self-rated health, including weight-hip ratio, height-adjusted weight.

Our study findings have strong implications for public health interventions. Given the strong association between self-rated health with BMI and weight, these measures could be a better option as the indicators to improve the self-perceptions of health among specific social and economic subgroups of the Chinese populations, hence maximize the effectiveness of public health interventions towards obesity, diabetes and other metabolic diseases.

\section{Acknowledgements \\ We are grateful for Clinical Trial Service Unit and Epidemiological Studies Unit (CTSU), Nuffield Department of Population Health, University of Oxford, Oxford, UK for providing the baseline data. \\ Funding \\ The authors declare no funding source. \\ Availability of data and material \\ The dataset supporting the conclusions of this article is available in the China Kadoorie Biobank http://www.ckbiobank.org/site/Data+Access}

\section{Authors' contribution}

TK and ZYX made the statistical analysis and drafted the manuscript; LCY revised the article for important intellectual content and wrote part of the article. All authors read and approved the final manuscript.

\section{Competing interests}

The authors declare that they have no competing interests.

\section{Consent for publication}

Not applicable.

\section{Ethics approval and consent to participate}

Ethical approval was obtained from the University of Oxford, the China National Center for Disease Control and Prevention (CDC) and the institutional review boards at the local CDCs in the 10 regions (Qingdao CDC, Heilongjiang CDC, Hainan CDC, Jiangsu CDC, Guangxi CDC, Sichuan CDC, Gansu CDC, Henan CDC, Zhejiang CDC, Hunan CDC) before the start of the survey. Written informed consent was obtained from all participants.

\section{Publisher's Note}

Springer Nature remains neutral with regard to jurisdictional claims in published maps and institutional affiliations.

\section{Author details}

'Department of Global Health, Peking University, Beijing, China. ${ }^{2}$ Institute for Medical Humanities, Peking University, Beijing, China. ${ }^{3}$ School of Public Health, Peking University, Beijing, China. 
Received: 25 June 2016 Accepted: 8 April 2017

\section{Published online: 13 April 2017}

\section{References}

1. Martikainen MG, Aromaa A, Heliovaara M, Dlaukka T, Knekt P, Maatele J, Lahelma E. Reliability of perceived health by sex and age. Soc Sci Med. 1999;48:1117-22.

2. Mossey J, Shapiro E. Self-rated health: a predictor of mortality among the elderly. Am J Public Health. 1982;72(8):800-8.

3. Idler EL, Benyamini Y. Self-rated health and mortality: a review of twenty-seven community studies. Journal of Health \& Social Behavior. 1997;38(1):21-37.

4. Roberts G. Age effects and health appraisal: A meta-analysis. J Gerontol B Psychol Sci Soc Sci. 1999:54B:S24-30.

5. Ross CE, Mirowsky J. Does employment affect health? J Health Soc Behav. 1995:36:230-43.

6. Popham F, Gray L, Bambra C, et al. Employment status and the prevalence of poor self-rated health. Findings from UK individual-level repeated crosssectional data from 1978 to 2004. BMJ Open. 2012;2(6):1285-95.

7. Jonnalagadda SS, Diwan S. Health behaviors, chronic disease prevalence and self-rated health of older Asian Indian immigrants in the U.S. J Immigr Health. 2005;7(2):75-83.

8. Farmer NM, Ferraro KF. Distress and perceived health: mechanisms of health decline. J Health Soc Behav. 1997;38(3):298-311.

9. Imai K, Gregg EW, Chen YJ, et al. The Association of BMI With Functional Status and Self-rated Health in US Adults. Obesity. 2008;16(2):402-8.

10. Prosper $M H, M o c z u l s k i ~ V L$, Qureshi A. Obesity as a predictor of self-rated health. Am J Health Behav. 2009;33(3):319-29.

11. Herman KM, Hopman WM, Rosenberg MW. Erratum to: Self-rated health and life satisfaction among Canadian adults: associations of perceived weight status versus BMI. Qual Life Res. 2013;22(10):2693-705.

12. Cullinan J, Gillespie P. Does Overweight and Obesity Impact on Self-Rated Health? Evidence Using Instrumental Variables Ordered Probit Models. Health Economics. 2015;25(10):1341-8.

13. Marques-Vidal P, Ravasco P, Paccaud F. Differing trends in the association between obesity and self-reported health in Portugal and Switzerland. Data from national health surveys 1992-2007. BMC Public Health. 2011;12(1):1-8.

14. Park JH, Lee KS. Self-rated health and its determinants in Japan and South Korea. Public Health. 2013;127(9):834-43.

15. Ha Y, Park H. Association between Obesity and Self-Rated Health in Korean Males and Females. Journal of Korean Biological Nursing Science. 2012;14:3.

16. Gómez-Ambrosi J, Silva C, Galofré JC, et al. Body Adiposity and Type 2 Diabetes: Increased Risk With a High Body Fat Percentage Even Having a Normal BMI. Obesity. 2011;19(7):1439-44.

17. Burton RF. Waist circumference as an indicator of adiposity and the relevance of body height. Med Hypotheses. 2010;75(1):115-9.

18. Sarría A, Moreno LA, Garcí-Liop LA, et al. Body mass index, triceps skinfold and waist circumference in screening for adiposity in male children and adolescents. Acta Paediatr. 2001;90(4):387-92.

19. Feng Z, Wang WW, Jones K, et al. An exploratory multilevel analysis of income, income inequality and self-rated health of the elderly in China. Soc Sci Med. 2012;75(12):2481-92.

20. Luo J, Qu Z, Rockett I, et al. Employment status and self-rated health in north-western China. Public Health. 2010;124(3):174-9.

21. Liu G, Zhen Z. Sociodemographic Differentials of the Self-Rated Health of the Oldest-Old Chinese. Population Research \& Policy Review. 2004;23(2): 117-33.

22. Chen Z, Lee L, Chen J, et al. Cohort profile: the Kadoorie Study of Chronic Disease in China (KSCDC). Int J Epidemiol. 2005;34:1243-9.

23. Chen Z, Chen J, Collins R, et al. China Kadoorie Biobank of 0.5 million people: survey methods, baseline characteristics and long-term follow-up. Int J Epidemiol. 2011:40:1652-66.

24. Du H, Bennett D, Li L, et al. Physical activity and sedentary leisure time and their associations with BMI, waist circumference, and percentage body fat in 0.5 million adults: the China Kadoorie Biobank study. Am J Clin Nutr. 2013; 97(3):487-96.

25. Kotsopoulos J, Baer HJ, Tworoger SS, et al. Anthropometric Measures and Risk of Epithelial Ovarian Cancer: Results From the Nurses' Health Study. Obesity. 2010;18(8):1625-31.

26. Rimm EB, Stampfer MJ, Giovannucci E, et al. Body Size and Fat Distribution as Predictors of Coronary Heart Disease among Middle-aged and Older US Men. Am J Epidemiol. 1995;141(12):1117-27.
27. Wang A, Arah OA. Body Mass Index and Poor Self-Rated Health in 49 LowIncome and Middle-Income Countries, By Sex, 2002-2004. Prev Chronic Dis. 2014;12(8):E133.

28. Asfar T, Ahmad B, Rastam S, et al. Self-rated health and its determinants among adults in Syria: a model from the Middle East. BMC Public Health. 2006;7(17):1-9.

29. Miyatake N, Matsumoto S, Miyachi M, et al. Relationship between changes in body weight and waist circumference in Japanese. Environ Health Prev Med. 2007;12(5):220-3.

30. Puhl RM, Heuer CA. The Stigma of Obesity: A Review and Update. Obesity. 1930:17(5):941-64.

31. Brewis AA, Hruschka DJ, Wutich A. Vulnerability to fat-stigma in women's everyday relationships. Soc Sci Med. 2011;73:491-7.

\section{Submit your next manuscript to BioMed Central and we will help you at every step:}

- We accept pre-submission inquiries

- Our selector tool helps you to find the most relevant journal

- We provide round the clock customer support

- Convenient online submission

- Thorough peer review

- Inclusion in PubMed and all major indexing services

- Maximum visibility for your research

Submit your manuscript at www.biomedcentral.com/submit
) Biomed Central 